\section{Simulating the binomial distribution with a software quincunx}

\author{
STEPHEN MADIGAN \\ University of Southern California \\ Los Angeles, California
}

The software quincunx is a graphics-based simulation of the binomial distribution designed as a teaching aid for statistics and probability instruction. The user can define the binomial probability, number of trials, and number of repetitions. The program graphically simulates the action of a mechanical quincunx and provides a summary of observed and expected data.

In this paper, I describe a graphics-based program that simulates a sequence of Bernoulli trials and the binomial distribution of outcomes. The program represents a software version of Galton's quincunx. Readers may be familiar with various mechanical versions of this device. The program incorporates its essential features (see Figure 1). A ball is introduced into the vertical shaft at the top and moves downward, being deflected to the left or right at each vertex. Galton's original device used lead shot and an array of pins projecting from a board; the name quincunx-according to the dictionary, an ornamental arrangement of five bushes-apparently referred to the placement of the pins. (See Stigler, 1986, pp. 275-281, for an interesting account of Galton's invention and use of the quincunx, as well as for drawings and photographs of the original device.)

Compared to simple mechanical versions, a software quincunx has the advantage of allowing control of the binomial probability, number of trials, and number of repetitions. The program can be useful for instruction in statistics and data analysis. We have found it especially effective by initially using a small number of trials (three, for example); students then readily grasp the "number of paths" principle.

Running a simulation. The program starts with a brief description of the quincunx and then asks for specification of parameters, as is illustrated in Figure 2. The user can enter a binomial probability of from .10 to .90 , which is the probability of the simulated ball's moving to the right at each choice-point. The number of trials (1-8) is

Correspondence should be addressed to Stephen Madigan, Department of Psychology, University of Southern California, Los Angeles, CA 90089 MC 1061. selected next, and then the number of repetitions $(10-1,000)$. While the simulation is running, there are six active function keys on the keyboard. These can be used to speed up or slow down the simulated motion, to add or remove sound effects, to pause and resume, and to terminate the simulation.

Program output. During program execution, the user sees a display like that shown in Figure 1, with a simulated ball moving through the quincunx. The display constantly increments the number of repetitions completed as well as the frequencies of outcomes along the base of the quincunx.

When the simulation is finished, a display such as the one shown in Figure 3 appears. Binomial probabilities and obtained and expected frequencies are displayed. The same information is presented in a bar graph as well. A chi-square goodness of fit test is also reported. Any of the display screens can be printed with Shift-Prtsc.

System requirements. The program will run on any PC-compatible system with $256 \mathrm{~K}$ or more of RAM and a CGA or higher video card. A math coprocessor is optional but highly desirable, especially for 8088 machines-if program execution time for a large simulation (for example, eight trials and 1,000 repetitions) is to be kept reasonable.

Software system. The program was developed with the Microsoft Basic Compiler 6.0. The RND function with seeding by the TIMER statement is used to produce each trial outcome. As Lordahl (1988) notes, this appears to produce satisfactory results for a one-variable classification of frequencies. An AUTOEXEC.BAT file installs the graphics print utility and then starts the program. This utility produces Epson-compatible compressed print. (If desired, the DOS GRAPHICS.COM file can be substituted for it.)

Availability. The program is available from the author at no cost. Send a 5.25-in. disk, a return address label, and a diskette mailer; or contact the author via BitNet at MCDOWD@NUNKI.USC.EDU.

\section{REFERENCES}

LORDahl, D. S. (1988). Repairing the Microsoft BASIC RND function. Behavioral Research Methods, Instruments, \& Computers, 20, 221-223.

Stigler, S. M. (1986). The history of statistics. Cambridge, MA: Harvard University Press/Belknap Press. 


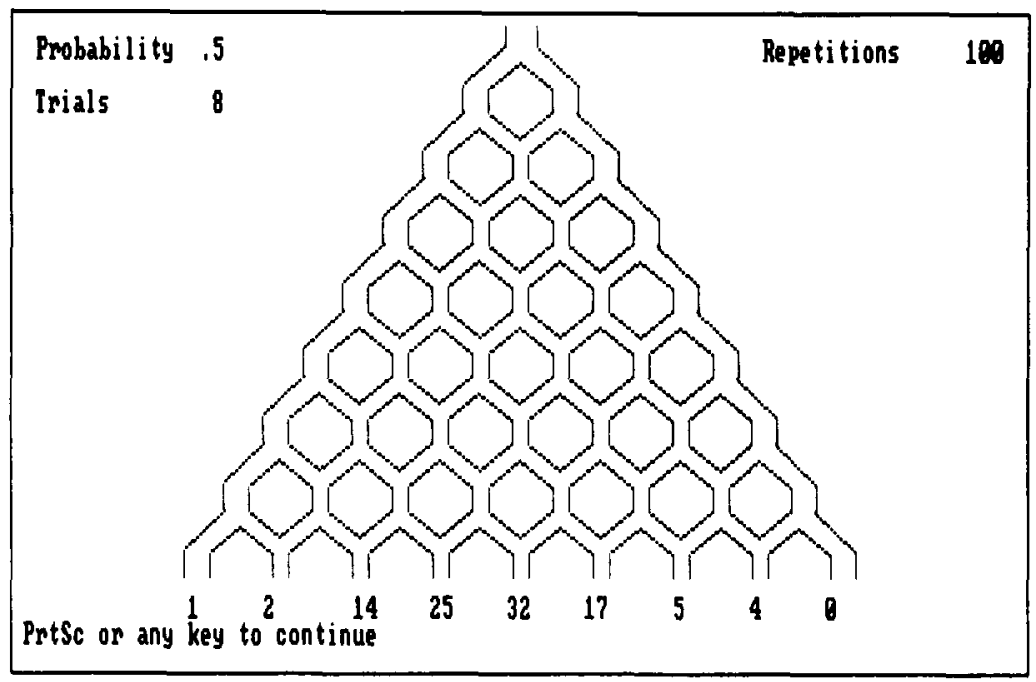

Figure 1. The software quincunx display for eight trials.

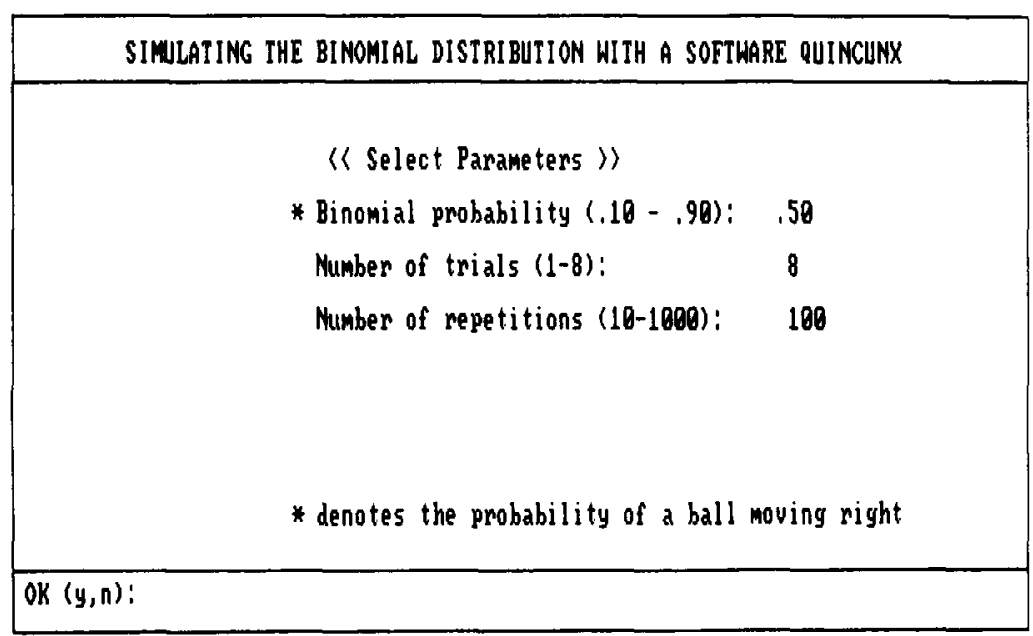

Figure 2. Simulation parameter definition.

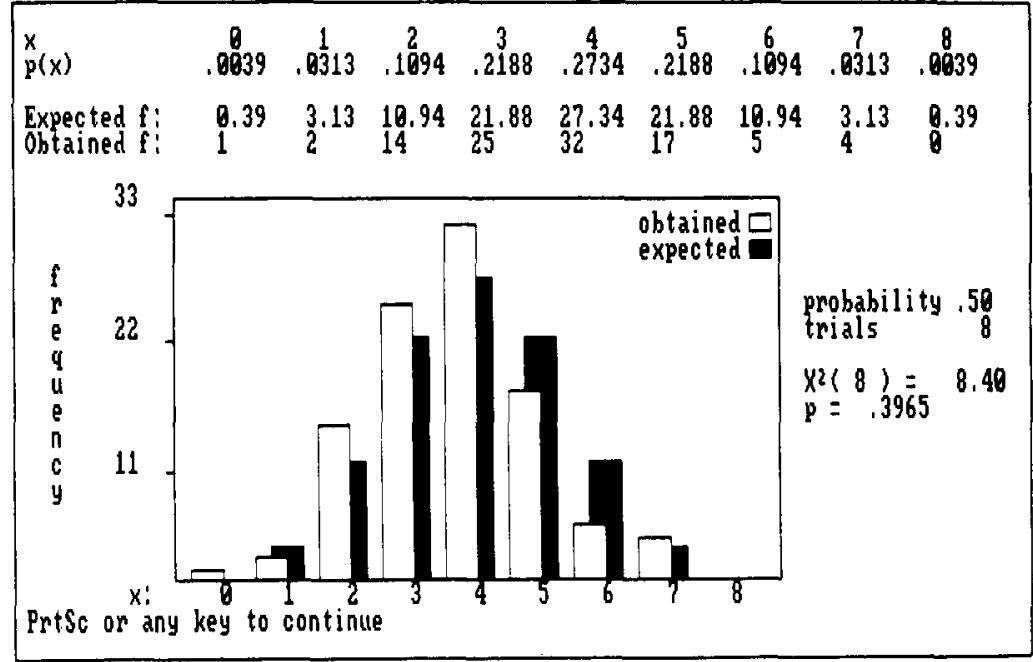

Figure 3. Summary of observed and expected frequencies. 